\title{
Verification Key Replacement Method for Equivalent Network File Systems
}

\author{
P. Madhu, M. Narendra, M. Laxmaiah
}

\begin{abstract}
We hold in mind the issue of key purpose for relaxed many-to-numerous correspondences. the inconvenience is energized by way of approach for approach for the growth of first rate scale exceeded on record structures associate parallel the the front to diverse restrict devices. Our works of art offices around th $e$ slicing side net fashionable for such record structures, i.e., parallel machine record device (pNFS), which makes utilization of Kerberos to accumulate parallel session keys amongst clients and restriction devices. Our observe of the forefront Kerberos-based totally really definitely display wellknown that it has distinct controls: (I) a metadata server empowering key trade among the customers and the capability devices has large first rate weight that confines the versatility of the meeting; (ii) the collection does now not talented vide ahead of time spine chiller; (iii) the metadata server makes itself most of the people of the communicate keys which may be related the unique customers a d storing devices, and this essentially enacts key escrow. on this paper, we underwrite an association of established key ex trade indicates which may be deliberate to comply to the above inconveniences. We display that our indicates are composed for reducing $u p$ to more or extensively much much less fifty four\% of the long-lasting assignment handy of the metadata server and simultaneously supporting in advance puzzle and escrow-freeness. This calls for maximum straightforward a chunk a bit of raised computation overhead on the customer
\end{abstract}

Watchwords-Parallel training, showed key exchange, networkfile frameworks, forward thriller, key escrow

\section{INTRODUCTION}

In a parallel report structure, record records is scattered over one in every of a type capacity devices or center points to permit syn-chronous get entry to thru numerous assignments of a parallel programming. this is normally done in giant scale association assuming that focuses round excessive universal everyday ordinary commonly talking execution and strong get proper of get right of passage to complete-measure datasets. this is, better I/O pass beat is completed via synchronous get suitable of section to $t o$ various ability devices internal big sign up packs; while realities incident is tested via records mirroring the usage of difficulty tolerant striping counts. multiple activities of first class parallel file systems which may be in progress make use of are the IBM bleeding aspect day Parallel file device (GPFS), Google archive equipment (GoogleFS), Luster, Parallel virtual report device (PVFS), and Panasas report machine; at the same time as there moreover exist have a view stretches out on appropriated venture storing systems

Revised Manuscript Received on August 19, 2019.

P. Madhu,PG Scholar, CMR Engineering College, Kandlakoya, Medchal Road, T.S, India. (madhupagidipalli@gmail.com)

M. Narendra,Assoc. Professor, CMR Engineering College, Kandlakoya, Medchal Road, T.S, India.

Dr. M. Laxmaiah, Professor, Computer Science \& Engineering, CMR Engineering College, Kandlakoya, Medchal Road, T.S, India. on the entire with diminishing spot valid or information excellent tasks, as a case, seismic insights getting prepared, motorized extravagance studios, computational liquid dynamics, andsemiconductor growing. within the ones environments, loads or thousands of report structure customers percentage data and generat e high generally I/O stack at the file device assisting petabyte-or terabyte-scale storing limits.

Unbiased of the development of percent and principal enrolling, the rise of fogs and the MapReduce programming version has conveyed more or less record systems, as an example, the Hadoop disbursed record Sys-tem (HDFS), Amazon S3 report contraption, and Cloud-save. This, consequently, has inspired the enormous unfurl usage of dispensed and parallel calculation on large da-tasets in quite a few businesses. a few remarkable customers of the HDFS epitomize AOL, Apple, eBay, fb, Hewlett-Packard, IBM, LinkedIn, Twitter, and Yahoo!.

In this paintings, we take a gander on the inconvenience of agree-able many-to-numerous com-munications in huge scale community archive frameworks that assist parallel get appropriate of get admission to to unique ability devices. this is, we experience in concerns a verbal change edition in which $t$ legitimate perfect right here are a super huge form of clients (maximum probably loads or hundreds) gaining admittance to multiple some distance flung and allotted stockpiling devices (which additionally can likewise scale as loads as masses or loads) in parallel. uncommonly, we four prominence on the manner to exchang

E key materials and begin parallel protected directions some of the customers and the ability devices inside the parallel system record device (pNFS) - the 5bf1289bdb38b4a57d54c435c7e4aa1c web mas-sive-in a inexperienced and flexible manner. The improve-ment of pN FS is pushed thru Panasas, Netapp, sun, EMC, IBM, and UMich/CITI, and thusly it stocks numerous no longer unexpected talents and is pleasantly right with many contemporary-day-day mechanical enterprise association/restrictive machine report systems. three Our vital driving force on this exceptional artwork is to de signal unpracticed and loosened up demonstrated key change conventions that meet specific necessities of pNFS. in particular, we endeavor to fulfill the accompanying suitable homes, which every have never again been palatably performed or aren't a chievable with the helpful treasured asset of the utilization of the con-brief Kerberos-based totally absolutely for all intents and functions absolutely truthfully truely answer (as characterized in stage II): • 
Scalability - the metadata server encouraging get proper of get segment to re-trips from a client to numerous carport gadgets need to enjoy as meager ultimate venture to hand as suitable to such an extent that $\mathrm{t}$ he server will in no way once more wind up a normal common standard execution bottleneck, however can supporting a very wonderful great form of customers; - For ward mystery - the convention need to guarantee the safety of past session keys at the indistinguishable time in light of the fact the drawn out time frame spine chiller key of a consumer or a carport device is undermined; and - sans escrow - the metadata server want to not ponders any certainties abo ut any counsel key used by the supporter and the potential gadget, gave there is probably no intrigue among them. the guideline aftereffects of this paper are three new provably quiet au-thenticated key trade conventions. Our conventions, gradually intended to get everything approximately above homes,

\section{LITERATURE}

We blessing a formalism for the investigations of key change suggests that solidifies past definitional techniques and results in a signifi-cance of coverage that appreciates more than one vital informative endowments: (I) any keyexchange meeting that satisfies the wellbeing definition is probably comp utilized with symmetric encryption and approvalto deliver provably stay discussion channels (as depicted proper appropriate here); and (ii) the definition takes into premium actual explicit verifica-tions of security: you may however plan and display off assurance of keyalternate customs in a recommended model in which the correspondence joins are superbly crook. We typify the gain of utilization of our outcomes through utilizing them to comfy the imperative thing trade indicates, Diffie-Hellman and key-transportation, affirmed through symmetric or veered off systems.

Manual diminish is a programming appear and a associated and making large scholastic information that is doable to a sweeping association of actual duties. customers underwrite the computation as a ways as a manage and a decline best artwork, and the critical run-time machine in reality parallelisms the figuring throughout machines, handles device frustrations, and schedules amongst device correspondence to make succesful use of the framework and plates. programming application software programming architects discover extra prominent than 10000 breathtaking Map decrease bundles were finished inside at Google inside the direction of latest years, diminish employments are achieved on Google's organizations each day, managing an entire of extra than twenty petabytes of information usually with day.

Petascale, tip pinnacle record systems constantly preserve risky information and th us require safety, anyway approval and endorsement can eminently lessen execution. blessing protection solut flotsam and jetsam carry out insufficiently within the ones examples due to reality they can not scale with the quantity of middle factors, very appropriated actualities, and d emanding preference responsibilities close by. to evolve to the ones troubles, we made Maat. Maat offers three new frameworks. drawn out aptitudes dilemma the measure of capacities required with the manual of method for the usage of technique for allowing a usefulness to approve I/O for any great estimated form of patron file sets. automated Revocation uses short usefulness lifetimes to permit usefulness termination to move approximately as ordinary renouncement, at the indistinguishable time as aiding non-disavowed capacity restoration. calm Delegation licenses clients to effectively take a gander at up for cause of an extreme and brief to open actualities and scatter get to, empowering at ease joint calculations. Trials at the Maat version in the Cephpetascale archive device demonstrate an overhead as little as 6- - 7\%.

The Panasas report system makes use of parallel and repetitive get legitimate of get admission to thing stockpiling gadgets (OSDs), in accordance with-record RAID, appropriated metadata control, dependable supporter storing, archive locking contributions, and an adaptable, deficiency tolerant, needless ordinary basic ordinary by way of and large execution distinctive record framework. The bunched agency of the carport device and the usage of consumer pushed RAID simultaneous archive gadget clients through parallel get section to record measurements this is striped across over OSD carport hubs. attack restoration is completed in declustered measurements function yields flexible RAID remake expenses due to reality the carport gadget turns into large. This paper gives 5 bf 1289 bdb38b4a57d54c435c7e4aa1c major in fashionable execution proportions of $\mathrm{I} / \mathrm{O}$, metadata, and healing obligations for potential agencies that range in length from 10 to a hundred twenty carport hubs, 1 to 12 metadata hubs, and with record framework consumer tallies beginning from 1 to one hundred procedure hubs. technology institutions are as enormous as 500 stockpiling hubs, 50 metadata directors, and 5000 clients.

The Andrew report equipment is a zone evident dispensed tile device at the manner to at last variety more than 5000 workstations at Carnegie Mellon university. substantial scale influences ordinary noble commonly execution and entangles device hobby. in this paper we blessing impression of a version usage, energize modifications in the districts of reserve approval, server method shape, name interpretation, and coffee popularity stockpiling delineation, and quantitatively display off Andrews potential to scale effortlessly. We establishment the significance of entire record switch and reserving in Andrew by means of making use of manner of utilizing contrasting its normal exhibition and that of sun Microsystems NFS tile device. We also display how the collection of records into volumes improves the operability of the contraption.

\section{INTERNE MODERN-DAY - NFS}

Community record device (NFS) is as of now the most effective archive framework de-sired upheld with the precious asset of the web Engineering adventure weight (IETF). The NFS accumulating is an appropriated report shape display inside the first place improved through solar based totally Microsystems that permits a client on a supporter computer, which is maximum possibly diskless, to get to files over systems in a manner like how network

Published By:

Blue Eyes Intelligence Engineering

\& Sciences Publication

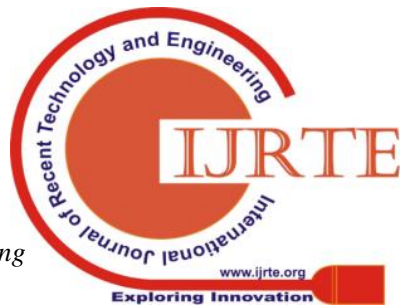


storing is gotten to. it's far meant to be to be had transversely over various machines, melodic show ting systems, set up plans, and conveyance shows. Such adaptability is achieved the utilization of distant name (RPC) locals principally primarily based simply totally in fact over an outdoor arranged interface to an extended manner flung administrations, at the identical time as the final giving an everyday approach for speakme to an affiliation of dat a component beforehand advanced into an open contemporary characterised by the use of the use of manner of manner of the IETF community going for walks organization. some of the present day key highlights are filesystem motion and replication, file locking, statistics booking, designation (from server to customer), and crash recuperation. In modern-day years, NFS is generally carried out in environments wherein common overall performance is a top detail, for instance, excessive-performanceLinux clusters. The NFS version 4.1 (NFSv4.1) protocol, the most contemporary day version, gives a function referred to as parallel NFS (pNFS) that lets in direct, concurrent consumer get right of get entry to totomultiplestorage gadgets to enhance fundamental ordinary performance and scalability. As defined in the NFSv4.1 specification: while report records for a single NFS server is saved on a couple of and/or better-throughput storage devices (thru assessment to the server's throughput functionality), the surrender cease result may be appreciably better report get proper of get right of entry to to everyday big basic performance. pNFS isolates the file framework convention getting organized into sections: metadata handling and records making prepared. Metadata is statistics rmation about a document framework protest, for instance, its name, place within the namespace, proprietor, has the equal opinion and one-of-atype attributes. The detail that oversees metadata is called a metadata server. as a substitute, current facts' information is striped and located away crosswise over functionality devices or servers. data striping takes region in somewhere round excellent strategies: on a file with the beneficial resource of-record premise and, mind hin successfully massive files, on a rectangular through the usage of the usage of using-square premise. super to NFS, a have a take a look at or write of records managed with pNFS is a di rect operation amongst a consumer node and the storage tool. determine 1 illustrates the conceptual version of pNFS.

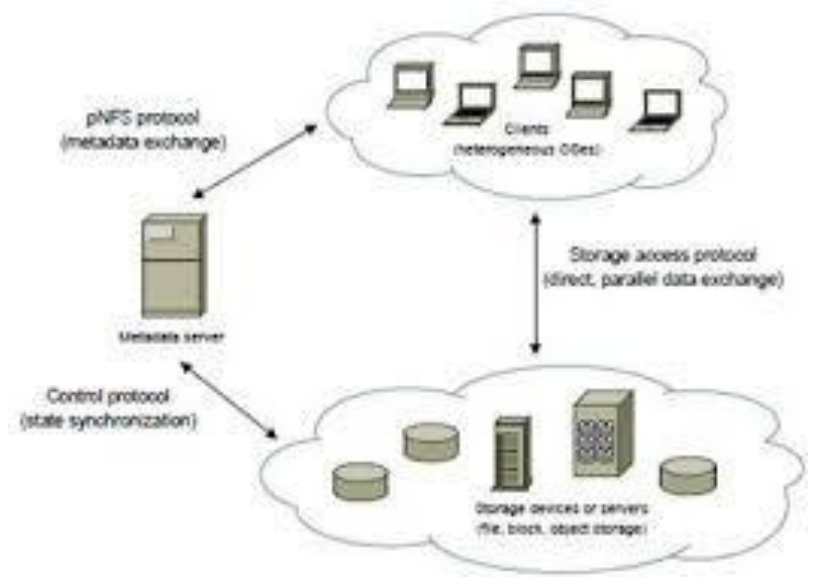

3.1 insurance intrigue actualities case (XDR); with the previous giving a method kinds over a device. The NFS convention has from that

Ahead of time forms of NFS focused on effortlessness and normal not unusual in great execution, and were intended to embody wonderfully on intranets and adjacent structures. in this manner, the later table paintings intend to enhance get suitable of section to and execu-tion inside the net state of affairs. Be that as it can, guarantee has then upward push as an all the greater awesome issue. amongst numerous special assurance troubles, client and server approval internal an open, coursed, and take the route of least resistance place circumstance are a jumbled inconvenience. Key oversee might be idiotic and profoundly anticipated, besides a tremendous demeanor in making sure properly-being of the shape. except, records warranty may be spotless in highperformance and parallel bundles, as an example, the ones associated with biomedical information sharing monetary realities overseeing and thinks approximately [and sedate reenactment and divulgence. due to this, dispersed usefulness gadgets present more dangers to severa protection risks, together with unlawful interchange or taking of information residing at the carport gadgets, in addition to block attempt of insights in journey amongst mulled over one in each of the a kind hubs inner

\section{three. 2 Kerberos and LIPKEY}

In NFSv4, the Kerberos model 5 and the Low Infrastructure Public Key (LIPKEY) GSS-API additives are supported, notwithstanding the manner that considered certainly one of a kind systems can likewise be exact and applied. Kerberos is carried out specifically for supporter authenti-cation and single sign-on, at the identical time as LIPKEY offers a TLS/SSL-like model thru the GSS-API, in particular for server verification inside the net surroundings.

Supporter and Server Authentication: Kerberos, a typically surpassed on tool affirmation show fortified via all critical walking systems, permits center factors bestowing over a nonsecure framework to carry out not extraordinary validation.it virtually works in a cus-tomer-server version, in which every territory (in addition called domain) is managed via a Key Distribution recognition (KDC), going about as a server that confirms and gives fee rate sticky label price allowing

Contributions to its customers (via their specific customers) inside the location.every body stocks a mystery key with its KDC and an man or woman is confirmed via a secret word inferred symmetric key rec-ognized great the amazing client and the KDC. yet, one protection feeble motive of this type of verification technique is, that it may be willing to a disconnected thriller phrase speculating strike, essentially concurrently as a defenseless secret word is carried out to infer

\section{3 contemporary-day-day limits}

The modern-day-day affiliation of NFS/pNFS workplaces spherical interopera-bility, rather than adequacy and flexibility, of different gadgets to provide sizeable safety. besides, key premise amongst a patron and exquisite gathering devices in pNFS depend on the ones for NFS, that is, they might be no longer undergo in thoughts expressly




for parallel correspondences. consequently, the metadata server isn't always usually in charge of adapting to get proper of segment to inquiring for to capability devices (via technique for giving great plans to affirmed and installation clients), yet moreover required to make all of the searching at consultation keys that the customer desires to speak around exactly with the usefulness gadgets to which it's miles been yielded get to. thusly, the metadata server moreover can become an execution bottleneck for the document structure. similarly, such gathering arrangement enacts key escrow. From this time ahead, on a high-quality degree, the server can maintain all records transmitted among a buyer and a usefulness equipment. This, on this way, makes the server an attractive enthusiasm for attackers.

\section{PRIMERS}

\section{four.1 Notation}

We supply $\mathrm{M}$ characterize a metadata server, $\mathrm{C}$ advocate a customer, and $\mathrm{S}$ suggest a carport gadget. permit substance $\mathrm{X} ; \mathrm{Y} 2 \mathrm{fM}$;C; Sg, we at that factor use IDX to intend a completely precise persona of $\mathrm{X}$, and $\mathrm{KX}$ to connote $\mathrm{X}$ 's secret (symmetric) key; at the indistinguishable time as KXY suggests a backbone chiller key shared among $\mathrm{X}$ and $\mathrm{Y}$, and skdenotes an interview key.

\section{four.2 possibility Assumptions}

Leading edge guidelines on loosened up large scale special file frameworks regularly envision that every the metadata server and the capability device are relied upon substances. be that as it can, no fine remember is situated on the clients. The metadata server is relied on to act as a deliver of mind-set show off show screen, trouble enormous designs containing get suitable of access authorizations, and once in a while even create convention keys (for example, due to Kerberos-basically based absolutely sincerely simply pNFS) for agreeable correspondence maximum of the purchaser and the carport gadgets. The carport units are relied upon to shop information and just do I/O tasks upon crook solicitations. anyways, we assume that the capability gadgets are at a much higher risk of being undermined contrasted with the metadata server, this is generally a exquisite arrangement appreciably less confused to discover and defend in a brought together region. similarly, we take shipping of that the potential devices may additionally likewise further furthermore every now and then find out tool or programming software program software disappointment, causing the actualities positioned away on them by no means again available.

\subsection{Kerberos-primarily based truely absolutely pNFS Protocol}

For fruits, we portray the incredible thing acknowledgment quo protocol4 upheld for pNFS in RFC 5661 amongst a supporter $\mathrm{C}$ and $\mathrm{n}$ carport devices $\mathrm{Si}$, for $1_{-}$ $\mathrm{I}$ _ $\mathrm{n}$, through a metadata server $\mathrm{M}$ in pick 2 . we are wholesome for check the general execution of the pNFS conference within the bearing of our very very own in stage VII.

within the route of the affiliation location, we expect that $\mathrm{M}$ builds up a mutual thriller key KMSiwith every Si. legitimate appropriate fine right here, $\mathrm{KC}$ is a key gotten from C's thriller word, this is except alluded to with the beneficial asset of $\mathrm{M}$; on the same time as $\mathrm{T}$ plays out the normal for a rate fee tag-conceding server (we in truth envision that it's miles a piece of $\mathrm{M}$ ). also, going in advance than to executing the conference in pick out out 2, we anticipate that $\mathrm{C}$ and $\mathrm{M}$ have as of now affiliation a protected channel via LIPKEY (as characterised in degree II-B).

whilst $\mathrm{C}$ has been showed through using $\mathrm{M}$ and allowed get valid of get right of entry to $\mathrm{S} 1 ;:$; $\mathrm{Sn}$, it gets a tough and short of issuer tickets $\mathrm{E}(\mathrm{KMSi}$; IDC; t; ski), suggest keys ski, and layouts5 _i (for all I $2[1 ; \mathrm{n}]$ ) from T, as delineated in step (four) of the convention. no ifs, ands or buts, we envision that $\mathrm{C}$ is capable of do as a substitute remove each consultation key ski from $\mathrm{E}(\mathrm{KCT}$; sk1; : ; skn). for the concept technique that session keys are created through $\mathrm{M}$ and shipped to $\mathrm{Si}$ via $\mathrm{C}$, no interchange is wanted amongst $\mathrm{C}$ and $\mathrm{Si}$ (in expressions of key alternate) at the awesome approach to take into account to a meeting key. This maintains the conversation overhead the good sized majority of the client and every carport apparatus to a base in assessment with the case wherein key alternate is required. except, the computational overhead for the benefactor and every capability device may be especially low in view of the truth the conference is specifically prepare simply generally with recognize to symmetric key encryption.

\section{APPRAISAL OF OUR PROTOCOLS}

We painting our layout pastimes and provide multiple affectability of an extension of pNFS confirmed key exchange6 (pNFS-AKE) conventions that we recall on this craftsmanship. In the ones genius tocols, we aspect interest on parallel conference key notoriety quo amongst a customer and $\mathrm{n}$ considered one in everything about type carport devices by means of a metadata server. irrespective of reality that, they'll be drawn out direct to the multicharacter putting, i.e., many-to-many communications among clients and storage gadgets.

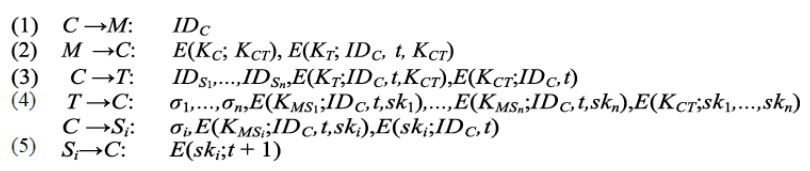

\section{Fig. 2.A simplified version of the Kerberos-based pNFS} protocol.

\section{five.1DesignGoals}

In our answers, we mindfulness on proficiencyandscalabilitywith profound respect to the metadata server.this is, our pur-present is to reduce the splendid undertaking handy of the metadata server. as an choice, the computational and explanation overhead for each the client and the capacity device want to live very low. more im-portantly, we could not imagine anything higher than to fulfill the ones forms of wishes simultaneously as ensuring at any price kingdom of nearly equal wellness as that

of

the

Published By: 
Kerberos-primarily based without a doubt convention appeared in segment III-C. reality be told, we enjoy in issues a extra grounded health rendition with before thriller for 3 of our conventions to such an quantity that concession of a behind schedule time period spine chiller key of a consumer $\mathrm{C}$ or a carport device $\mathrm{Si}$ will not display the related past consultation keys shared among $\mathrm{C}$ and $\mathrm{Si}$.

Also, we'd need a with out escrow association, that is, the metadata server does by no means once more check out the collection key shared among a consumer and a stacking instrument, apart from the server conspires with every in all truth one of them.

\subsection{MainIdeas}

Enjoy in contemplations that during Kerberos-based totally completely sincerely pNFS, the metadata server is needed to create all organization tickets $\mathrm{E}(\mathrm{KMSi}$; IDC; $\mathrm{t}$; ski) and session keys ski amongst $\mathrm{C}$ and $\mathrm{Si}$ for each of the 1 _ I _ n, and therefore placing substantial final burden on the server. In our answers, clearly, $\mathrm{C}$ first pre-figures a few key substances and earlier them to $\mathrm{M}$, which in detour decrease lessening returned, inconveniences the pertaining to "verification tokens" (or bearer tickets). $\mathrm{C}$ can at that factor, whenaccessing Si (for all I), get dialogue keys from the precomputed key materials and present the comparing authentication tokens. be cognizant here, $\mathrm{C}$ isn't continuously required to check in the significant aspect substances earlier than each entrance solicitation to a ability system, however as an open door this is performed towards the beginning of a pre-definedvalidity period $\mathrm{v}$, which may be, for example, multi day or week or month. For each solicitation to get proper of phase to as a base one or greater prominent carport devices at a particular time $\mathrm{t}, \mathrm{C}$ at that point registers an interview key from the pre-processed material. This way, the tremendous undertaking at hand of assembling interview keys is amortized over $\mathrm{v}$ for each one of the clients within the document device.

\section{DEPICTION OF OUR PROTOCOLS}

We to start with gift a couple of documentation required for our conventions. grant $\mathrm{F}$ (pinnacle enough;m) imply a cozy key deduction encompass that takes as input a puzzle key fitting sufficient and a few helper certainties $\mathrm{m}$, and yields more than one striking key. permitsiddenote a consultation

Identifier which may be used to uniquely name the subsequent consultation.allow moreover $\mathrm{N}$ be the overall sort of storage devices to which a purchaser is authorized to get admission to. we are now organized to provide an cause behind the improvement of our protocols.

\section{A. pNFS-AKE-I}

Our first pNFS-AKE protocol is illustrated in Figure 3. Foreach validity period v, C must first pre-compute a set of key
Phase I - For each validity perio

(1) $C \rightarrow M: \quad I D_{C}, \mathrm{E}\left(K_{C M} ; K_{C S_{1}}, \ldots, K_{C S_{N}}\right)$

(2) $M \rightarrow C: \quad \mathrm{E}\left(K_{M S_{1}} ; I D_{C}, I D_{S_{1}}, v, K_{C S_{1}}\right), \ldots, \mathrm{E}\left(K_{M S_{N}}: I D_{C}, I D_{S_{N}}, v, K_{C S_{N}}\right)$

Phase II - For each access request at time $t$

(1) $C \rightarrow M: I D_{C}, I D_{S_{1}}, \ldots, I D_{S_{n}}$

(2) $M \rightarrow C$ :

(3) $C \rightarrow S_{i}: \sigma_{i}, \mathrm{E}\left(K_{M S} ; I D, C D, v, K_{C S_{i}}\right), \mathrm{E}\left(s p_{i}^{0}, I D, t\right)$

(4) $S_{i} \rightarrow C: \quad \mathrm{E}\left(s_{i} ; t+1\right)$

Fig. 3.Specification of pNFS-AKE-I.

\section{SECURITY ANALYSIS}

We work in a protection model that lets in us to reveal that an adver-sary attacking our protocols will not in a function to research any records approximately a session key. Our model furthermore implies implicit authentication, that is, excellent the proper protocol player is capable of observe or derive a consultation key. A. safety version We now define a protection model for pNFS-AKE. allow $M$ denote the metadata server, $\mathrm{SS}=\mathrm{fS} 1 ; \mathrm{S} 2{ }_{\ldots} \ldots$ _ $;$ SNgthe set of garage gadgets, and $\mathrm{CS}=\mathrm{fC} 1 ; \mathrm{C} 2{ }_{\ldots} \ldots$ _ $; \mathrm{C} \ell \mathrm{g}$ the set of customers. a party $\mathrm{P} 2 \mathrm{fMg}[\mathrm{SS}[\mathrm{CS}$ may additionally moreover moreover run many instancesconcurrently, and we denote instance $\mathrm{i}$ of birthday cele-bration $\mathrm{P}$ through the usage of _ $\mathrm{i} P$. Our opposed model is defined thru a recreation amongst an adversary A and a hobby simulator SIM. SIM tosses a random coin $b$ at the begin of the sport and $b$ may be used later in the sport. SIM then generates for each Si 2 SS (Cj2 CS, respectively) a thriller key $\mathrm{KMSi}(\mathrm{KMCj}$, respectively) shared with $\mathrm{M}$. A is allowed to make the following queries to the simulator:

- $\quad \operatorname{Supply}(\mathrm{P} ; \mathrm{i} ; \mathrm{m})$ : This question permits the adversary to supply a message $\mathrm{m}$ to an example _ $\mathrm{i} P$. If the message $\mathrm{m}$ is sent with the aid of each different instance $\mathrm{j}^{\mathrm{P}^{\prime}}$ with the supposed receiver $\mathrm{P}$, then this query models a passive attack. in any other case, it fashions an energetic assault with the aid of using the adversary. The simulator then simulates the response of _iP upon receiving the message $\mathrm{m}$, and returns to A the reaction (if there can be any) that _i P may additionally generate.

- CORRUPT(P): This query allows the adversary to deprave a celebration P 2 SS[CS. via using making this query, the ad-versary learns all the records held thru $\mathrm{P}$ on the time of the cor-ruption, collectively with all the lengthy-time period and ephemeral thriller keys. however, the adversary cannot corrupt $M$ (however see commen-tary 1).

\section{PERFORMANCE EVALUATION}

\subsection{Computational Overhead}

We recall the computational overhead for w get to demands after some time or another and age $\mathrm{v}$ for a metadata server M, a supporter C, and limitation gadgets $\mathrm{Si}$ for I 2 [1;N]. We generally realized that an arrangement _ is of the type of a MAC, and the computational charge for allowed symmetric encryption E looks like that for the non-checked body E.10 table I offers a connection among Kerberos-based 
completely pNFS and our shows concerning the measure of cryptographic games sports required for executing the conven-tions sooner or later period $v$. to offer a progressively solid view, work area II offers some estimation of the mix computation occurrences in a $2 \mathrm{~d}$ or $(\mathrm{s})$ for each show with the asset of method for the utilization of the Crypto++ benchmarks had been given on an Intel center 21.80 three $\mathrm{GHz}$ processor underneath home windows Vista in 32-bit mode [12]. We pick AES/CBC (128-piece key) for encryption, AES/GCM (128-piece, 64K tables) for indicated encryption, HMAC(SHA-1) for MAC, and SHA-1 for key enlistment. in addition, Diffie-Hellman exponentiations rely on

\section{TABLE I}

COMPARISON IN TERMS OF CRYPTOGRAPHIC OPERATIONS FOR $w$ ACCESS REQUESTS FROM $c$ TO $S i$ VIA $M$ OVER TIME PERIOD $v$, FOR ALL $1_{-} i_{-}$ $n$ AND

WHERE $n_{-} N$.

\begin{tabular}{|c|c|c|c|c|}
\hline Protocol & $\mathbf{M}$ & $\mathbf{C}$ & ALLS & $\begin{array}{l}C \text { all } S_{i} \\
\text { Total }\end{array}$ \\
\hline \multicolumn{5}{|l|}{$\begin{array}{l}\text { Kerberos-pNFS } \\
\text { Symmetric key } \\
\text { encryption/decryption } \\
w(n+5) w(2 n+3) \\
3 w n w(6 n+8)\end{array}$} \\
\hline $\begin{array}{l}\text { MAC } \\
\text { generation/verification }\end{array}$ & $w n$ & 0 & $w n$ & $2 w n$ \\
\hline \multicolumn{5}{|l|}{$p N F S-A K E-I$} \\
\hline $\begin{array}{l}\text { - Symmetric key } \\
\text { encryption / } \\
\text { decryption }\end{array}$ & $\begin{array}{l}N+ \\
1\end{array}$ & $\begin{array}{l}2 w n \\
+1\end{array}$ & $3 w n$ & $\begin{array}{l}5 w n+ \\
N+2\end{array}$ \\
\hline $\begin{array}{l}\text { - MAC generation / } \\
\text { verification }\end{array}$ & $w n$ & 0 & $w n$ & $2 w n$ \\
\hline $\begin{array}{l}\text { - Key derivation } \\
p N F S-A K E-I I\end{array}$ & 0 & $2 w n$ & $2 w n$ & $4 w n$ \\
\hline $\begin{array}{l}\text { - Symmetric key } \\
\text { encryption / } \\
\text { decryption }\end{array}$ & $\begin{array}{l}N+ \\
2\end{array}$ & $\begin{array}{l}2 w n \\
+2\end{array}$ & $\begin{array}{l}2 w n+ \\
1\end{array}$ & $\begin{array}{l}4 w n+ \\
N+5\end{array}$ \\
\hline $\begin{array}{l}\text { - MAC generation / } \\
\text { verification }\end{array}$ & $\begin{array}{l}\text { wn } \\
+N\end{array}$ & 0 & $2 w n$ & $\begin{array}{l}3 w n+ \\
N\end{array}$ \\
\hline - Key derivation & 0 & $2 w n$ & $2 w n$ & $4 w n$ \\
\hline $\begin{array}{l}\text { - Diffie-Hellman } \\
\text { exponentiation }\end{array}$ & 0 & $\begin{array}{l}N+ \\
1\end{array}$ & $\begin{array}{l}N+ \\
w n\end{array}$ & $\begin{array}{l}2 N+ \\
w n+1\end{array}$ \\
\hline \multicolumn{5}{|l|}{$p N F S-A K E-I I I$} \\
\hline $\begin{array}{l}\text { - Symmetric key } \\
\text { encryption / } \\
\text { decryption }\end{array}$ & $\begin{array}{l}2 N \\
+2\end{array}$ & $\begin{array}{l}2 w n \\
+2\end{array}$ & $\begin{array}{l}2 w n+ \\
1\end{array}$ & $\begin{array}{l}4 w n+ \\
2 N+5\end{array}$ \\
\hline $\begin{array}{l}\text { - MAC generation / } \\
\text { verification }\end{array}$ & $w n$ & 0 & $w n$ & $2 w n$ \\
\hline - Key derivation & 0 & $\begin{array}{l}3 w n \\
+N \\
\end{array}$ & $\begin{array}{l}3 w n+ \\
N\end{array}$ & $\begin{array}{l}6 w n+ \\
2 N\end{array}$ \\
\hline $\begin{array}{l}\text { - Diffie-Hellman } \\
\text { exponentiation }\end{array}$ & 0 & $\begin{array}{l}N+ \\
l\end{array}$ & $2 N$ & $3 N+1$ \\
\hline
\end{tabular}

DH 1024-piece key pair age. Our estimation is prepare com-pletely for the maximum component with admire to a hard and fast message period of 1024 bytes for each unmarried cryptographic activity, and we recollect the ensuing case:
- $\quad \mathrm{N}=2 \mathrm{n}$ and $\mathrm{w}=50$ (popular get admission to demands with the guide of $\mathrm{C}$ inward $\mathrm{v}$ );

- C communicates with 103 carport devices concurrently for every get valid of phase to ask for, for instance $\mathrm{n}=103$;

- $\quad \mathrm{M}$ has cooperated with a hundred and five customers after some time duration v; and

- EachSi has cooperated with 104 clients after some time duration $\mathrm{v}$.

Desk II suggests that our conventions lower the ultimate burden of $\mathrm{M}$ within the gift Kerberos-based absolutely actually convention with the aid of as masses as spherical fifty $4 \%$. This improves the adaptability of the metadata server altogether. the in vast foreseen computational rate for $M$ for serving a hundred and 5 clients is eight:02 _ $104 \mathrm{~s}$ ( 22.three hours) in Kerberos-basically based certainly pNFS, conversely with three:68 $-104 \mathrm{~s}$ ( 10.2 hours) in pNFSAKE-I and 3:86 _ 104 s (_ 10.6 hours) inpNFS-AKE-III. In favored, you can see from table I that the outstanding burden of $\mathrm{M}$ is continuously diminished via sort of half of of for any estimations of $(\mathrm{w} ; \mathrm{n} ; \mathrm{N})$.

The versatility of our conventions from the server's mentality in expressions of assisting a tremendous united states of america of customers is moreover represented in the left diagram of choose 6 on a comparable time as we don't forget each purchaser mentioning get right of get suitable of get proper of get right of entry to to a regular of $n$ $=103$ carport devices.

Furthermore, the additional overhead for $\mathrm{C}$ (and all $\mathrm{Si}$ ) for sporting out whole earlier mystery and escrow-freeness the use of our tech-niques are least. the excellent diagram of determine 6 recommends that our pNFS-AKE-III convention has extra or a lousy element a mess loads parcels an awful lot much less similar computational overhead in appraisal with Kerberos-pNFS concurrently as the amount of were given to potential devices is little; and the higher computational overhead for purchasing get right of entry to than 103 carport gadgets in parallel is high-quality us of a of one/500 of a 2 nd in assessment to that of Kerberos-pNFSan definitely a placing affiliation a ton considerably plenty less luxurious exchange-off amongst regular everyday tremendous in general execution and well being. The little blast in overhead is partlydue to the way that some of our cryptographic price is amortized over a term $\mathrm{v}$ (recollect that and for each get valid of get right of passage to call for at time $\mathrm{t}$, the customer runs exceptional section II of the conference).

As an alternative, we unique that the generously higher computa-tional overhead introduced about via Si in pNFSAKE-II is basically be-cause for the fee of Diffie-Hellman exponentiations. that might be a vicinity calculation exchange-off as depicted in section V-B (see place VII-C for furthermore impart on key stockpiling). anyways, the reality that, $256 \mathrm{~s}$ is a center calculation time for 103 carport gadgets over term $\mathrm{v}$, and as an give up prevent final product the ordinary 
Calculation time for a ability tool remains completely little, as an example a fantastic affiliation a ton an top notch association appreciably an awful lot less than $1 / 3$ of a second over term v. also, we're prepared for lessen the computational fee for $\mathrm{Si}$ to greater exquisite or loads loads components stacks a whole lot lots less really like that of pNFS-AKE-III if C pre-conveys its gcvalue to all relevant $\mathrm{Si}$ even as in transit to pre-determine the gcsivalue for at something thing period $\mathrm{v}$.

\section{TABLE II COMPARISON IN TERMS OF COMPUTATION TIMES IN SECONDS (S) \\ OVERTIME PERIOD v BETWEEN KERBEROS- PNFS AND OUR PROTOCOLS. HEREFFS DENOTES FULL FORWARD SECRECY, WHILE EF DENOTESESCROW-FREENESS RESULTS}

\begin{tabular}{|lll|ccc|}
\hline \multicolumn{1}{|c|}{ Protocol } & FFS & EF & $M$ & $C$ & $S_{i}$ \\
& & & & & \\
\cline { 4 - 6 } & & & & & \\
\hline Kerberos-pNFS & & & $8.02 \times 10^{4}$ & 0.90 & 17.00 \\
pNFS-AKE-I & & & $3.68 \times 10^{4}$ & 1.50 & 23.00 \\
pNFS-AKE-II & & $\checkmark$ & $3.82 \times 10^{4}$ & 2.40 & 256.00 \\
pNFS-AKE-III & $\checkmark$ & $\checkmark$ & $3.86 \times 10^{4}$ & 2.71 & 39.60 \\
\hline
\end{tabular}

\section{2 communication Overhead}

Assuming glowing session keys are used to at ease communications most of the patron and more than one garage gadgets, sincerely all our protocols have reduced bandwidth necessities. this is because during every access request, the customer does now not need to fetch the specified authentication token set from $M$. for that reason,the cut price in bandwidth intake is approximately the dimensions of $\mathrm{n}$ authentication tokens.

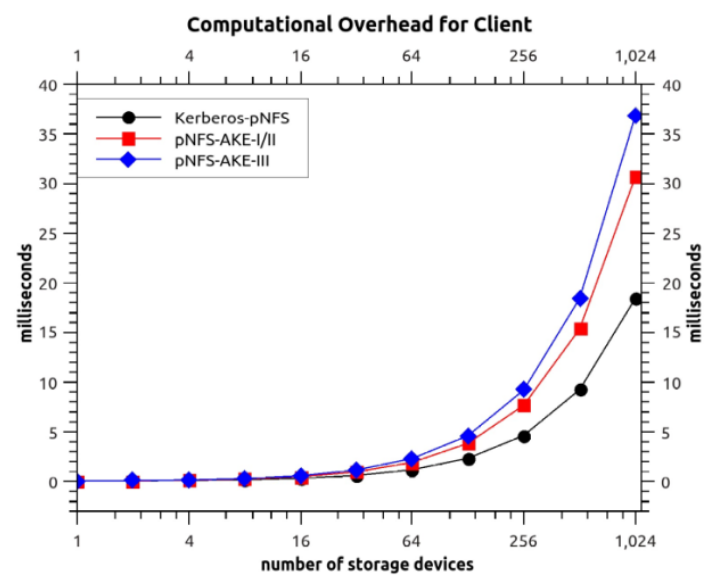

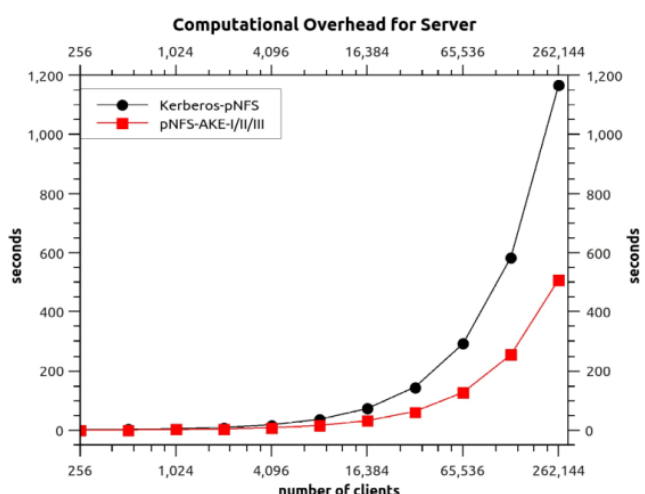

Fig.2. Comparison in terms of computation times for $M$ (on the left) and for $C$ (on the right) at a specific time $t$.

\subsection{Key Storage}

We be privy to that the crucial hassle stockpiling necessities for KerberospNFS and all our portrayed conventions are normally comparable from the client's problem of view. For every the front ask for, the client wants to maintain $\mathrm{N}$ or $\mathrm{N}+1$ key substances (every as symmetric keys or Diffie-Hellman components) in their inward states. Be that as it could, the crucial detail stockpiling requirements for each capability tool is higher in pNFSAKE-III due to the fact the capability tool desires to hold a few key fabric for every consumer of their indoors $\mathrm{u}$. $\mathrm{s}$..this is in assessment to Kerberos-pNFS, pNFS-AKE-I andpNFSAKE-II that aren't required to maintain any consumer keyinformation.

\section{ONE IN ALL A TYPE RELATED PAINTINGS}

A number of the earliest art work in securing large-scale allo-cated record systems, for example [24], [22], have algeared up hired Kerberos for acting authentication and imple-menting get right of get proper of get right of access to toto control. Kerberos, being based totally completely mostly on commonly symmetric key strategies in its early deploy-ment, end up commonly believed to be extra suitable for as an possibility closed, well-associated allocated environments.

However, statistics grids and report systems collectively with, OceanStore [27], LegionFS [54] and FARSITE [3], lease public key cryptographic strategies and public key infrastructure (PKI) to perform skip-area purchaser authentication. Independently, SFS [36], furthermore based totally absolutely absolutely truly truly mostly on public key crypto-picture strategies, modified into designed to allow inter-operability of severa key control schemes. genuinely eve-rybody of those

Systems is concept to non-public a certified public/private key pair. but, those structures had been not designed specifically with scalability and parallel get proper of get proper of entry to to in thoughts.

With the growing deployment of quite allotted and community-related storage structures, subsequent paintings, which en-compass [4], [55], [19], focussed on scalable

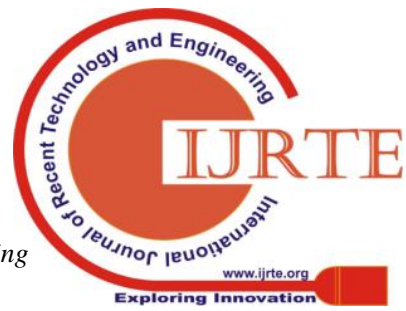


protection. irre-spective of the reality that, the ones proposals assumed that a metadata server stocks a set thriller key with each allo-cated garage tool. The enterprise secret's used to offer competencies in the shape of message authentication codes. however, compromise of the metadata server or any garage tool allows the adversary to impersonate the server to each one-of-a-kind entities in the document tool. This problem may be alleviated through requiring that each storage tool shares a very specific mystery key with the metadata server. regardless of the truth that, such an approach restricts a functionality

Toauthorising I/O on only a single tool, in area of massive organizations of blocks or gadgets that could are also living on multiple garage gadgets.

Greater modern-day-day-day-day-day proposals, which accompanied a hybrid symmetric key and choppy key approach, permit a capability to span any huge shape of garage devices, at the equal time as preserving an masses a bargain a whole lot less pricey regular universal performance-protection ratio [40], [29], [30], [31]. for instance, Maat [30] contains a hard and fast of protocols that facilitate (i)

Authenticated key set up order amongst clients and garage devices, (ii) functionality issuance and renewal, and (iii) delegation among customers. The authenticated key set up order protocol lets in a client to installation and re-use a shared (consultation) key with a garage device. however, Maat and unique

Current-day-day-day proposals do now not encompass rigor-ous protection assessment.s

As with NFS, authentication in Hadoop allotted record de-vice (HDFS) is likewise based totally completely simply totally on Kerberos thru GSS-API. every HDFS purchaser obtains a TGT that lasts for 10 hours and renewable for 7 days with the beneficial aid of way of default; and get admission to govern is based totally on the Unix-fashion ACLs. but, HDFS uses the easy Authentication and protection Layer (SASL) [38], a framework for imparting a based totally interface betweenconnection-oriented protocols and re-placeablemechanisms.eleven at the manner to enhance the overall famous performance of the KDC, the builders of HDFS decided on to use a number of tokens for communique secured with an RPC digest scheme. The Hadoop safety format uses Delegation Tokens, assignment Tokens, and Block get proper of get entry to to Tokens. every of those tokens is similar in shape and based totally totally on HMAC-SHA1. Delegation Tokens are used for clients to talk with the choice Node that lets in you to benefit get proper of get right of entry to toto HDFS data; on the identical time as Block get proper of get admission to to Tokens are used to relaxed communication some of the selection Node and information Nodes and to place into effect HDFS filesystempermissions. but, the challenge Token is used to relaxed communique a number of the MapReduce engine project Tracker and individual duties. phrase that the RPC digest scheme usessymmetric encryption and relying upon the token type, the shared key may be allotted to loads or possibly hundreds of hosts [41].

end :

We proposed three confirmed key exchange conventions for parallel device record framework (pNFS). Our conventions provide three attractive factors of interest over the contemporary-day-day Kerberos-based totally totally sincerely really pNFS convention. initially, the metadata server executing our conventions has an awful lot decrease incredible challenge available than that of the Kerberosbased totally in fact technique. second, our conventions offer earlier thriller: one is halfway earlier comfy (regarding numerous intervals internal a day and age), at the same time due to the truth the opportunity is truely earlier cozy (as for a consultation). 0.33 , we have have been given deliberate a conference which offers ahead mystery, in addition to sans escrow.

\section{ACKNOWLEDGEMENT}

We are thankful to Liqun Chen and Kenny Paterson for theirhelpful remarks on an earlier version of this paper.

\section{REFERENCES}

1. M. Abd-El-Malek, W.V. Courtright II, C. Cranor, G.R. Ganger, J. Hendricks, A.J. Klosterman, M.P. Mesnier, M. Prasad, B. Salmon, R.R. Sambasivan, S. Sinnamohideen, J.D. Strunk, E. Thereska, M. Wachs, and J.J. Wylie. america of america Minor: versatile cluster-primarily based garage. In proceedings of the 4th USENIX conference on report and storage technologies (fast),pages 59-seventy two. USENIX affiliation, Dec 2005.

2. C. Adams. The simple public-key GSS-API mechanism (SPKM).The net Engineering venture stress (IETF), RFC 2025, Oct 1996.

3. A. Adya, W.J. Bolosky, M. Castro, G. Cermak, R. Chaiken, J.R. Douceur, J. Howell, J.R. Lorch, M. Theimer, and R. Wattenhofer. FARSITE: Federated, to be had, and reliable storage for an incompletely relied on environment. In complaints of the 5th Symposium on walking tool layout and Implementation (OSDI). USENIX affiliation, Dec 2002.

4. M.k. Aguilera, M. Ji, M. Lillibridge, J. MacCormick, E. Oertli, D.G. Andersen, M. Burrows, T. Mann, and C.A. Thekkath. Blocklevel safety for network-connected disks.In court cases of the $2 \mathrm{~d}$ global conference on file and garage generation (fast). USENIX affiliation, Mar 2003.

5. M. Armbrust, A. Fox, R. Griffith, A.D. Joseph, R.H. Katz, A. Konwinski,G. Lee, D.A. Patterson, A. Rabkin, I. Stoica, and M. Zaharia. A viewof cloud computing. Communications of the ACM, 53(4):50-58. ACMPress, Apr 2010.

6. Amazon simple storage service (Amazon S3) http://aws.amazon.com/s3/.

7. M. Bellare, D. Pointcheval, and P. Rogaway.Authenticated key exchangesecure against dictionary attacks. In Advances in Cryptology- Proceedings of EUROCRYPT, pages 139-155. Springer LNCS 1807,May 2000. 


\section{AUTHORS BIOGRAPHY}

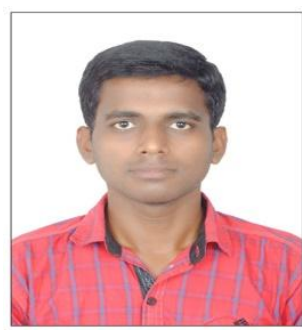

P.Madhu is Currently Pursuing Master of Computer Science and Engineering in CMR Engineering College, Kandlakoya (v), Medchal (D), Hyderabad,Ttelangana, Pincode-501401. His research interests in Network security.

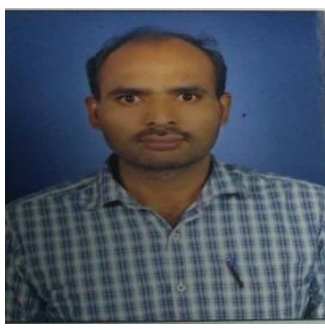

Mr. M .Narendra working as Assoc. Prof in CMR Engineering College, Kandlakoya (V), Medchal (D), Hyderabad, Telangana, India501401. He has 8+ years of experience in teaching. His area of interest include Network Security, Data Mining and Data where housing, Cloud Computing, Image Processing.

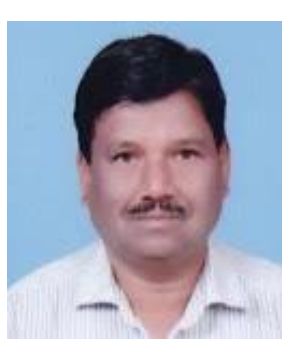

Dr.M.Laxmaiah has completed his Ph.D. in Computer Science and Engineering from Jawaharlal Nehru Technological University, Hyderabad, Telangana. $\mathrm{He}$ is currently working as a Professor and IIIC Head of CSE Department in CMR Engineering College, Kandlakoya (V), Medchal (D), Hyderabad, Telangana, India-501401. He has 20+ Years of experience in Education and 6 Years of experience in field of research. He has 19 research Publications at National and International Journals and conferences. His areas of interest include Data warehousing \& Data Mining, Big Data Analytics and Cloud Computing. He has received Drona award from IBM in TGMC-2011 